\title{
Comparative Study of Serologic Results Obtained by HI Test of Avian Influenza and Newcastle Disease Performed in Four Mexican Laboratories
}

\author{
Alberto Guadarrama ${ }^{1}$, Daniel Marrufo ${ }^{1}$, Eduardo Lucio ${ }^{1}$ and Lucía Elizabeth Cruz $^{2}$ \\ 1. Birds Technical Department, Investigación Aplicada S.A. de C.V., Tehuacan 75700, Puebla, Mexico \\ 2. Regulatory Affairs Department, Sanfer-Investigación Aplicada S.A. de C.V., Mexico City 01040, Mexico
}

\begin{abstract}
In the poultry industry, it is common to evaluate the humoral immune response associated to the vaccination calendar used for the different zootechnical purposes. When the vaccine against avian influenza and Newcastle disease currently used in a farm is going to be changed, an important parameter observed to choose a product over the others is based on the antibody titers reached by the application of the new vaccine. This study aimed to compare the serologic results obtained by hemagglutination inhibition (HI) test of avian influenza and Newcastle disease reported in four different national laboratories. One-day-old Ross broiler chickens were kept in Horsfall-Bauer isolation units and were vaccinated subcutaneously for the prevention of avian influenza and Newcastle disease. Then, serum of all the birds was extracted at three, six and seven weeks old and sent to four different national diagnostic laboratories, where HI test was performed for avian influenza and Newcastle disease. The treatments were designed in $4 \times 3$ factorial. Data showed significant statistical differences between laboratory results (up to six logarithms for influenza at six and seven weeks). This study confirms that the results of the HI test can vary from one laboratory to another, thus it is important to consider this, when the vaccines against avian influenza and Newcastle disease are evaluated at field.
\end{abstract}

Key words: Avian influenza, Newcastle disease, serology, hemagglutination inhibition test.

\section{Introduction}

The Newcastle disease is one of the most important infections in birds. The causal agent of Newcastle disease, virulent Newcastle disease virus (NDV), also known as avian paramyxovirus type 1, belongs to the Avulavirus genus within the Paramyxoviridae family. Virions have a single-stranded, negative-sense, non-segmented RNA genome that codes for six structural proteins: nucleoprotein (NP), phosphoprotein (P), matrix (M), fusion (F), hemagglutinin-neuraminidase (HN) and RNA polymerase (L) [1]. The hemagglutinin-neuraminidase and F glycoproteins allow NDV to attach and fuse to the host cell membranes, thereby initiating infection. Antibodies against hemagglutinin-neuraminidase and fusion neutralize the virus and are a primary

Corresponding author: Alberto Guadarrama, M.Sc., research field: agricultural sciences. component of vaccine-induced protection versus NDV [2]. Avian paramyxovirus 1 (APMV-1), synonymous with NDV, is a geographically widespread viral agent that infects wild, peri-domestic (living in or around human habitations) and domestic avian hosts [3]. It is distributed around the world and causes big economic losses in poultry [4]. The causal agent of the NDV is capable of infecting 240 bird species, and it spreads by direct contact with sick birds [5]. APMV-1 strains of lower virulence have also been detected in numerous species of wild birds which may or may not result in clinical disease [6]. Although some viruses may be differentially maintained in wild or domestic bird reservoirs, there is also evidence of viral transmission across the poultry-wild bird interface [7].

In addition to biosecurity and culling of infected birds, vaccination is a critical component to control Newcastle disease [8]. Flock immunity is another 
beneficial consequence of a successful vaccination program, as it provides some protection to sub-optimally vaccinated or unvaccinated birds in an otherwise well vaccinated flock. However, this outcome is only achieved with Newcastle disease when greater than $85 \%$ of the flock have hemagglutination inhibition (HI) antibody titers greater than $\log _{2}(8)$ after two vaccinations [9]. The NDV can be used as antigen in a wide variety of serologic tests, which allows the use of neutralization techniques or enzyme-linked immuno sorbent assay (ELISA) test and HI test to evaluate the antibody level in birds. Currently, the HI test is the most widely used test for antibody detection against NDV in birds [10].

The family Orthomyxoviridae contains four genera: influenza A, B, C and thogotovirus. Three different types (A, B and C) of influenza viruses are classified on the basis of antigenic differences among their nucleocapsid and matrix proteins. Influenza A viruses are further divided into subtypes based on the antigenic relationships of their hemagglutinin (HA) and neuraminidase (NA) surface glycoproteins [11].

The most virulent disease that affects highly susceptible birds can vary from a sudden death with few clinical signs, to a more characteristic disease with varied clinical signs with respiratory symptoms, such as ocular and nasal discharge, cough, dyspnea, swollen nasal passages or head, cyanosis on not feathered skin, wattle and comb, signs of incoordination, nervousness and diarrhea [10].

Because all influenza virus type A have similar antigens from the nucleocapsid and matrix, agar gel immuno-diffusion is used in order to detect antibodies against this antigens. For this test, concentrated virus preparations are used with one or both antigen types, but not all the birds species develop demonstrable antibodies. The HI test has also been used in routine diagnostic serology, even though it might not detect some specific infections because of the specificity of the hemagglutinin for the virus subtype [10].
Vaccination is being more commonly considered for use, because in some situations it could be a more cost effective control strategy as compared to the standard eradication through stamping out programs for the control of avian influenza [12]. Currently, extensive studies are being conducted to develop more effective vaccines to control avian influenza in addition to the traditional inactivated vaccine [13].

In the poultry industry, it is common to evaluate the humoral immune response associated to the vaccination calendar used for the different zootechnical purposes (breeders, layers and broilers). When the vaccine against avian influenza and Newcastle disease currently used in a farm is going to be changed, an important parameter observed to choose a product over the others is based on the antibody titers reached by the application of the new vaccine. However, these results can have significant differences according to the laboratory performing the test, the antigen used in vaccines and the antigen used in the HI test. It is important to consider this when assessment of vaccines and calendars are made in order to assure the results are reliable. Therefore, this study aimed to compare the serologic results obtained by $\mathrm{HI}$ test of avian influenza and Newcastle disease reported by four different national laboratories.

\section{Materials and Methods}

\subsection{Birds}

Thirteen one-day old broiler Ross breed chickens from a company in Cordoba, Veracruz were used. 2.5 $\mathrm{mL}$ of blood were extracted from the jugular of each bird every week. The samples were placed in BD vacutainer ${ }^{\circledR}$ tubes at room temperature for $12 \mathrm{~h}$. Once $1 \mathrm{~mL}$ of serum was obtained from each tube, they were collected in Crioval tubes for each group (52 tubes each sampling). A total of 208 samples were taken during the experiment.

The birds were placed since day of birth in a Horsfall-Bauer isolation unit with filtered air at negative pressure. They had free access to water and 
food and the temperature was regulated according to the age of the birds.

\subsection{Vaccine}

An emulsified vaccine containing avian influenza (PRONABIVE strain) and Newcastle (LaSota strain) antigens was used. The dose applied was $0.5 \mathrm{~mL}$ subcutaneously injected.

\subsection{Laboratories}

The samples were placed in different vials of 1,000 $\mu \mathrm{L}$, identified by group with consecutive numbers and sent to four national laboratories: Investigación Aplicada (IA), Diagnosticos Clinicos (AV), Universidad Nacional Autonoma de México (UN) and Biotecnologia Veterinaria (BI). These laboratories are certified by the Mexican Certification Entity (EMA).

\subsection{HI Tests}

HI test of avian influenza and Newcastle disease were performed for the samples of the serum obtained. The HI test for avian influenza was performed according to the procedure described in the OIE terrestrial manual [14]. The HI test for Newcastle disease was performed according to the procedure described in the Mexican official norm (NOM-052-ZOO-1995) [15] with the minimum requirements for vaccines employed in the prevention and control of Newcastle disease.

\subsection{Statistic Analysis}

The experiment design was random by factorial treatment $4 \times 3$ (Table 1 ). The birds were bled at 3-, 6and 7-week-old, with 13 repetitions.

\section{Results}

\subsection{HI Results of Newcastle Disease}

All four laboratories worked with the LaSota antigen. It can be observed that in the basal monitoring (first sampling), the results of each laboratory are very similar in number without any significant difference (Figs. 1 and 2). However, 23\% of the samples analyzed by the AV laboratory were positive to Newcastle disease, probably due to the fact that the birds are offspring of previously vaccinated birds. The highest parameters were reported by AV and UN laboratories and the lowest results by the $\mathrm{BI}$ laboratory. In this first sampling, it can be observed a seroconversion associated with the vaccination against Newcastle disease. In the last sampling (Fig. 3), it can be observed that the test performed in the AV laboratory shows differences up to four logarithms compared with the UN laboratory which obtained the highest titers from the four evaluated laboratories. Unlike the second monitoring, in this monitoring, the lowest titers were reported by the AV laboratory, which shows $61.5 \%$ of the serums with a negative titer.

\subsection{HI Results of Avian Influenza}

All four laboratories worked with PRONAVIBE antigen. The laboratory identified as UN, reported higher titers than the other laboratories. In this case, the results might be positive because the birds used for the experiment come from previously vaccinated mothers with avian influenza in the field (Fig. 4).

It can be observed that the laboratory identified as IA obtained the lowest result, and there are even

Table 1 Experiment design of the study.

\begin{tabular}{ll}
\hline Activity & Age of the birds \\
\hline Birds reception & Day of birth \\
First sample: ND and AI vaccine application & 3-week-old \\
Second sample & 6 week-old \\
Third sample & 7 week-old \\
\hline
\end{tabular}

AI: avian influenza; ND: Newcastle disease. 

Newcastle Disease Performed in Four Mexican Laboratories

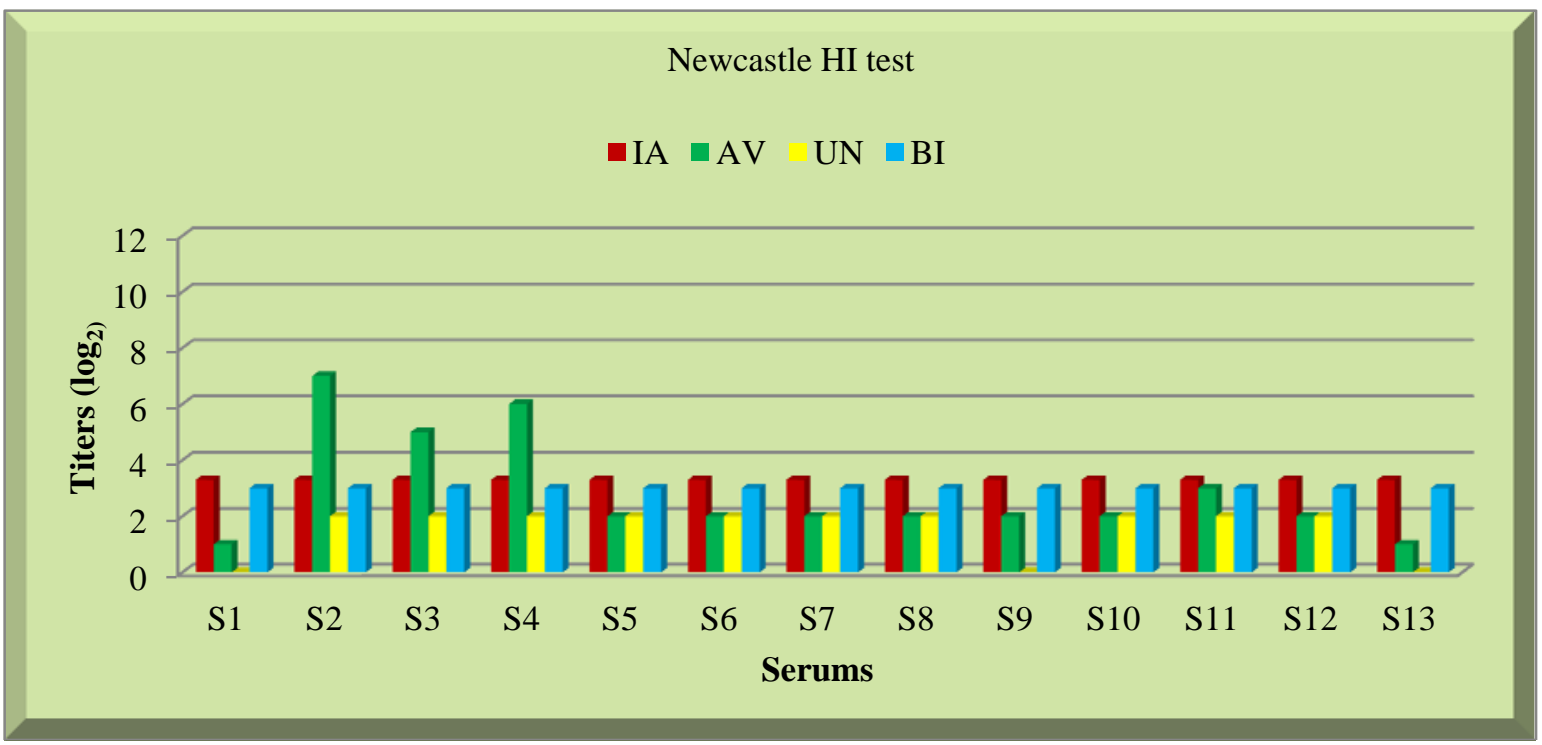

Fig. 1 HI test results of Newcastle of the first serologic sampling at 3-week-old.

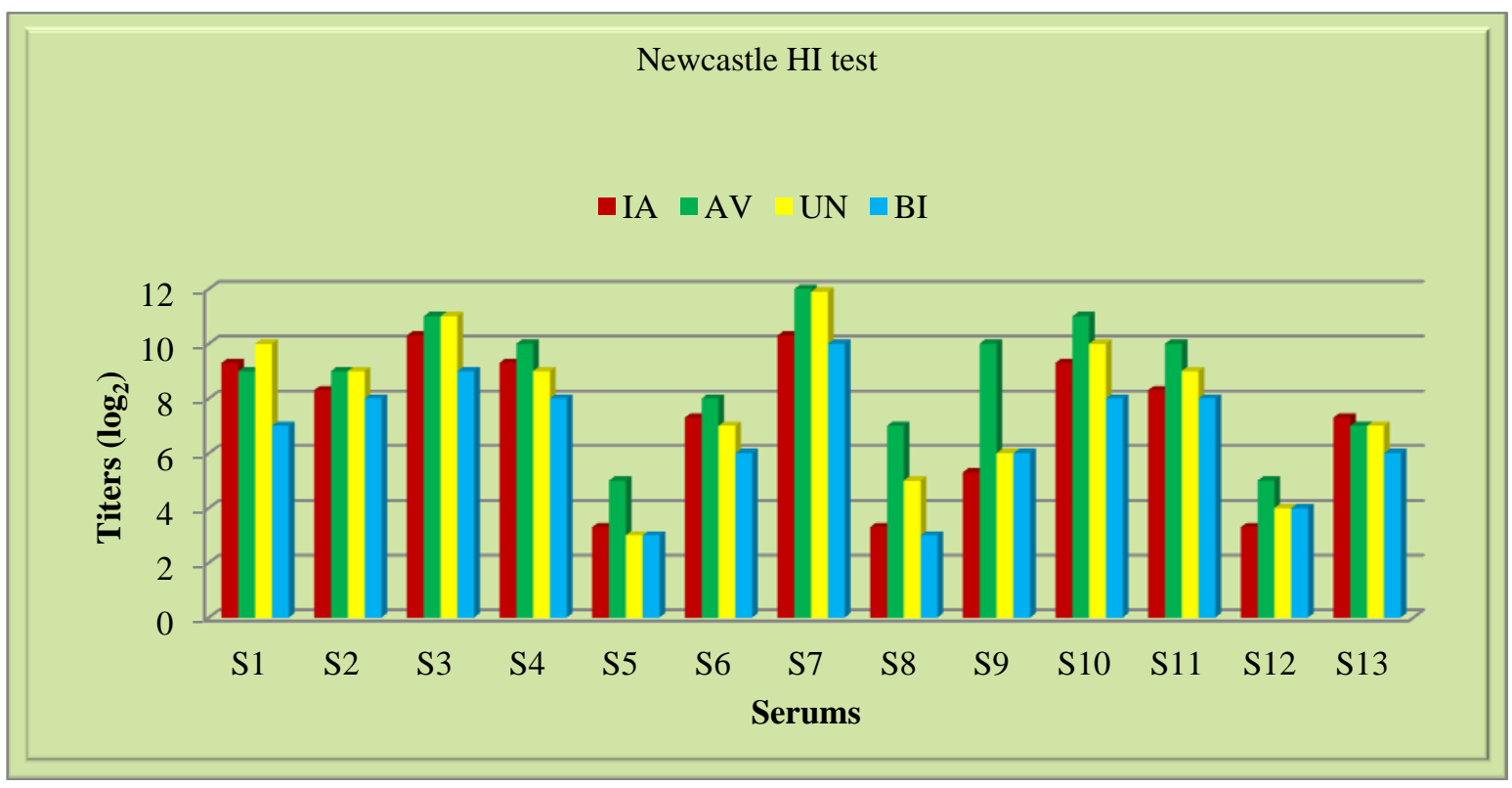

Fig. 2 HI test results of Newcastle of the second serologic sampling at 6-week-old.

differences of four logarithms in comparison with the results obtained by the other laboratories. A marked difference can be observed in avian influenza titers compared to those of Newcastle disease titers in the second monitoring. As well as in the second sampling, the highest titers were obtained by IA laboratory where $100 \%$ of the serums had higher titers. The lowest titers were reported by the AV laboratory in the same way as the second monitoring. A very marked variation is observed in titers reported by all four laboratories.

\section{Discussion}

The World Organization for Animal Health (OIE) mentions that the HI test for both the Newcastle disease and avian Influenza are widely used to determine the antibody titers for these diseases [10]. For this reason and due to the importance of this test, a comparison was performed where the results emitted by the laboratories were different for avian influenza 

Newcastle Disease Performed in Four Mexican Laboratories

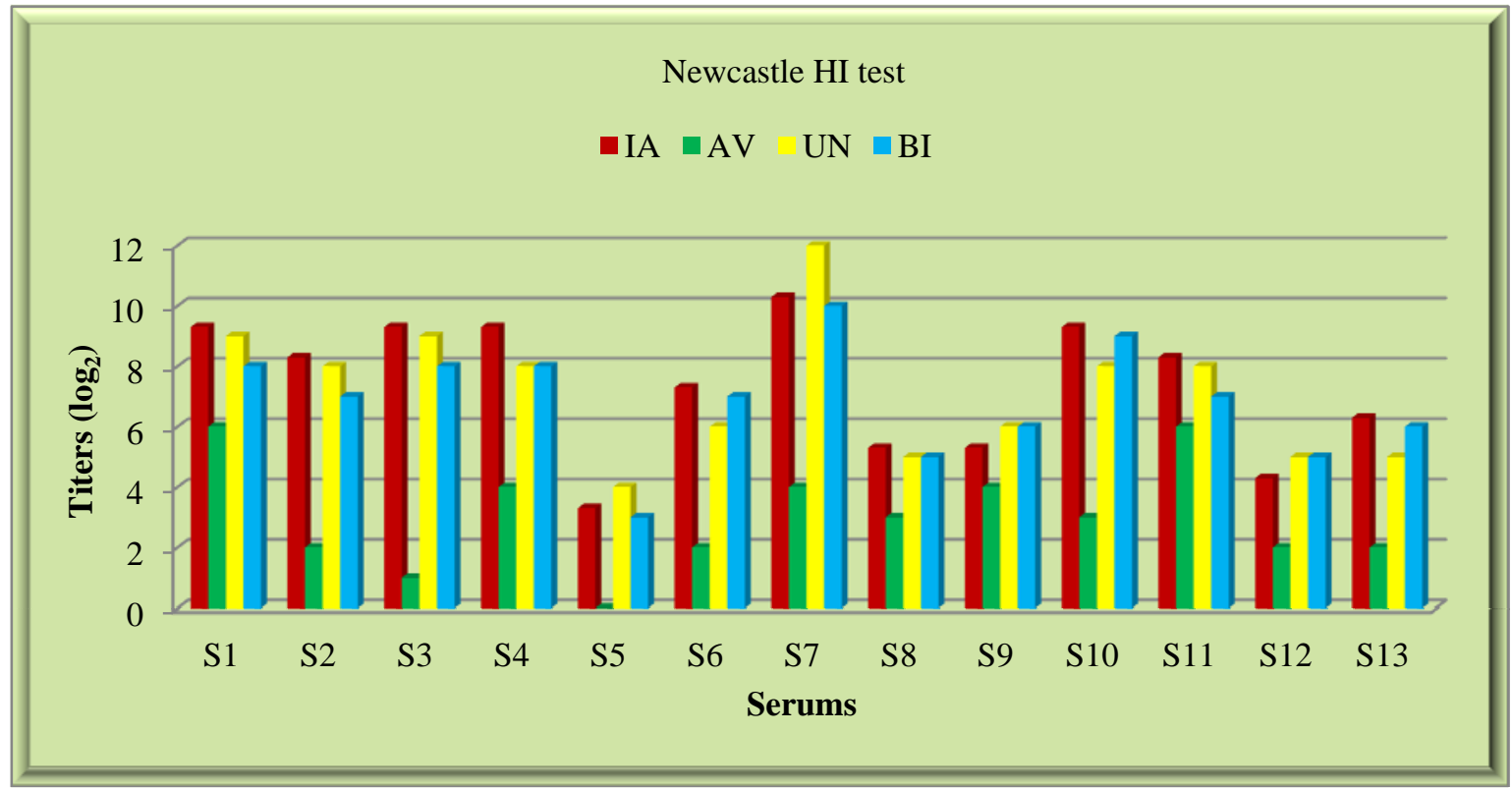

Fig. 3 HI test results of Newcastle of the third serologic sampling at 7-week-old.

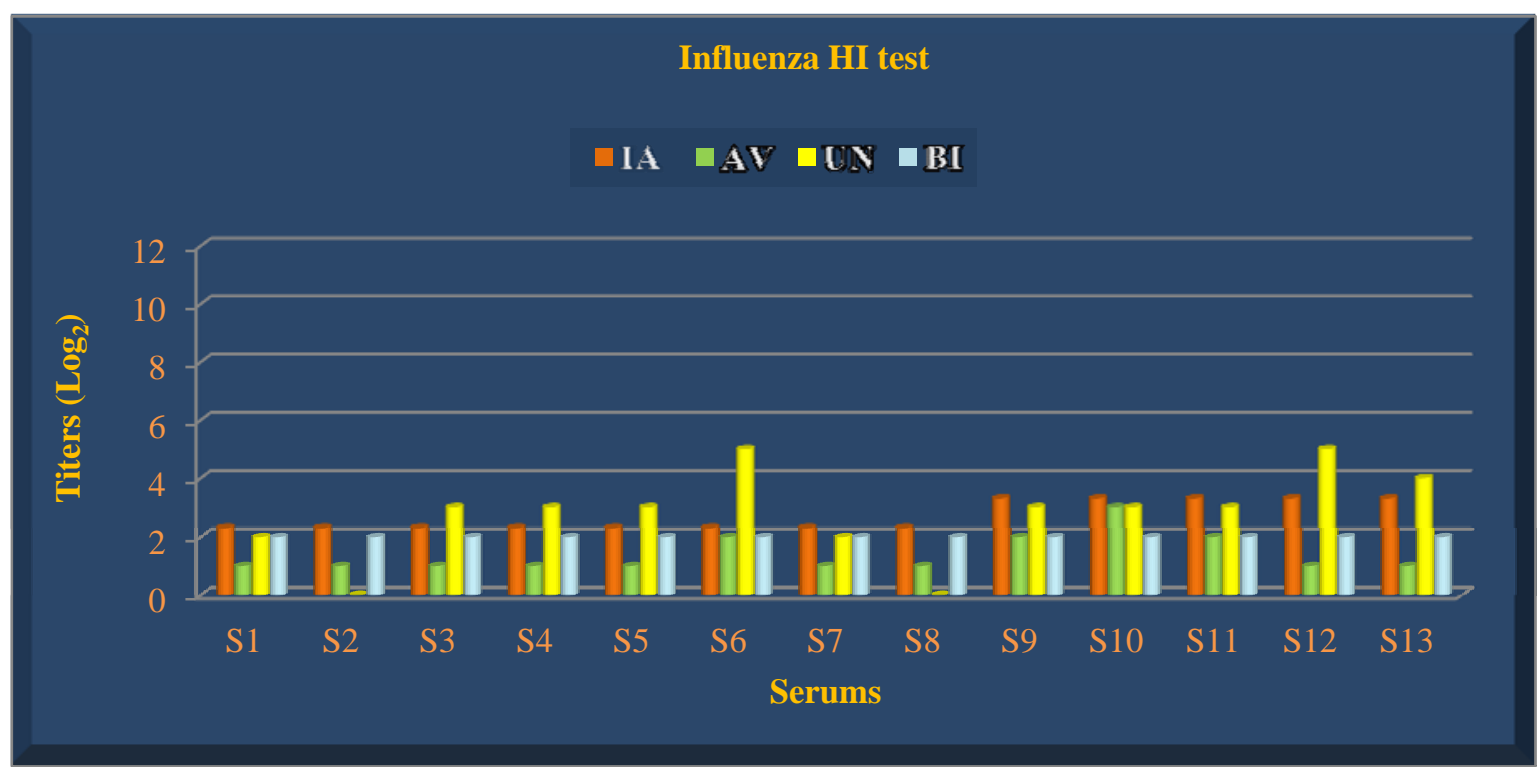

Fig. 4 HI test results of avian influenza of the first serologic sampling at 3-week-old.

in the second and third serologic sampling.

Valladares [16] mentioned that the HI test was widely used for the H5 subtype of avian influenza. Due to the fact that the antigenic properties of the hemagglutinin can be modified by site mutations, the homology between the antigen of the test and the field virus is very important to achieve an adequate level of antibody detection. In this study, the seroconversion was found for both avian influenza and Newcastle disease in vaccinated birds and detected for all four national laboratories. However, a difference was found in avian influenza results probably associated to the homology between the antigens of the test (Figs. 5 and 6).

For the Newcastle disease, Kapczynski and King [17] mentioned that field results suggest that only birds with $\mathrm{HI}$ titers greater than $\log _{2}(16)$ after multiple vaccinations will survive a NDV challenge 


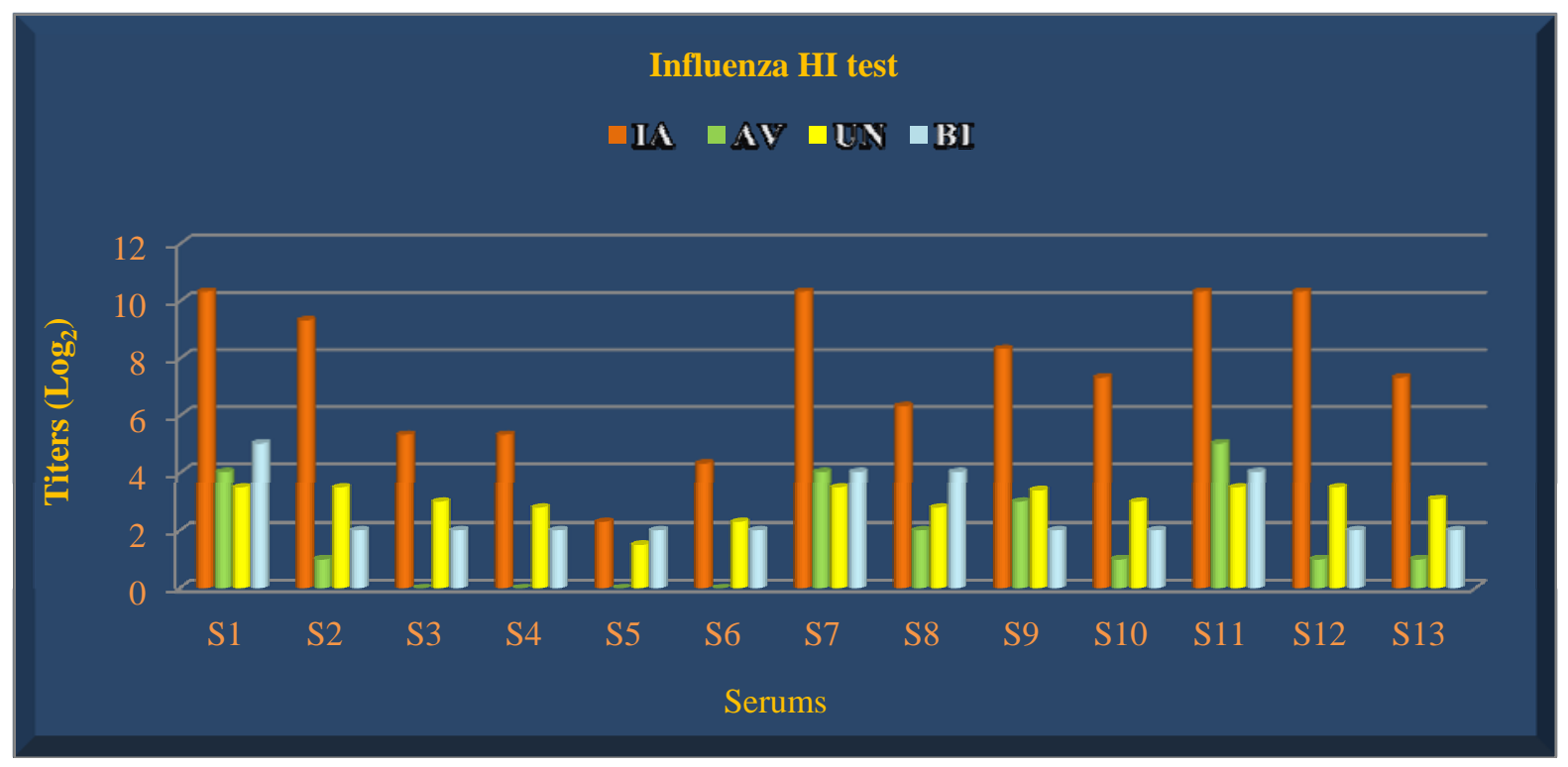

Fig. 5 HI test results of avian influenza of the second serologic sampling at 6-week-old.

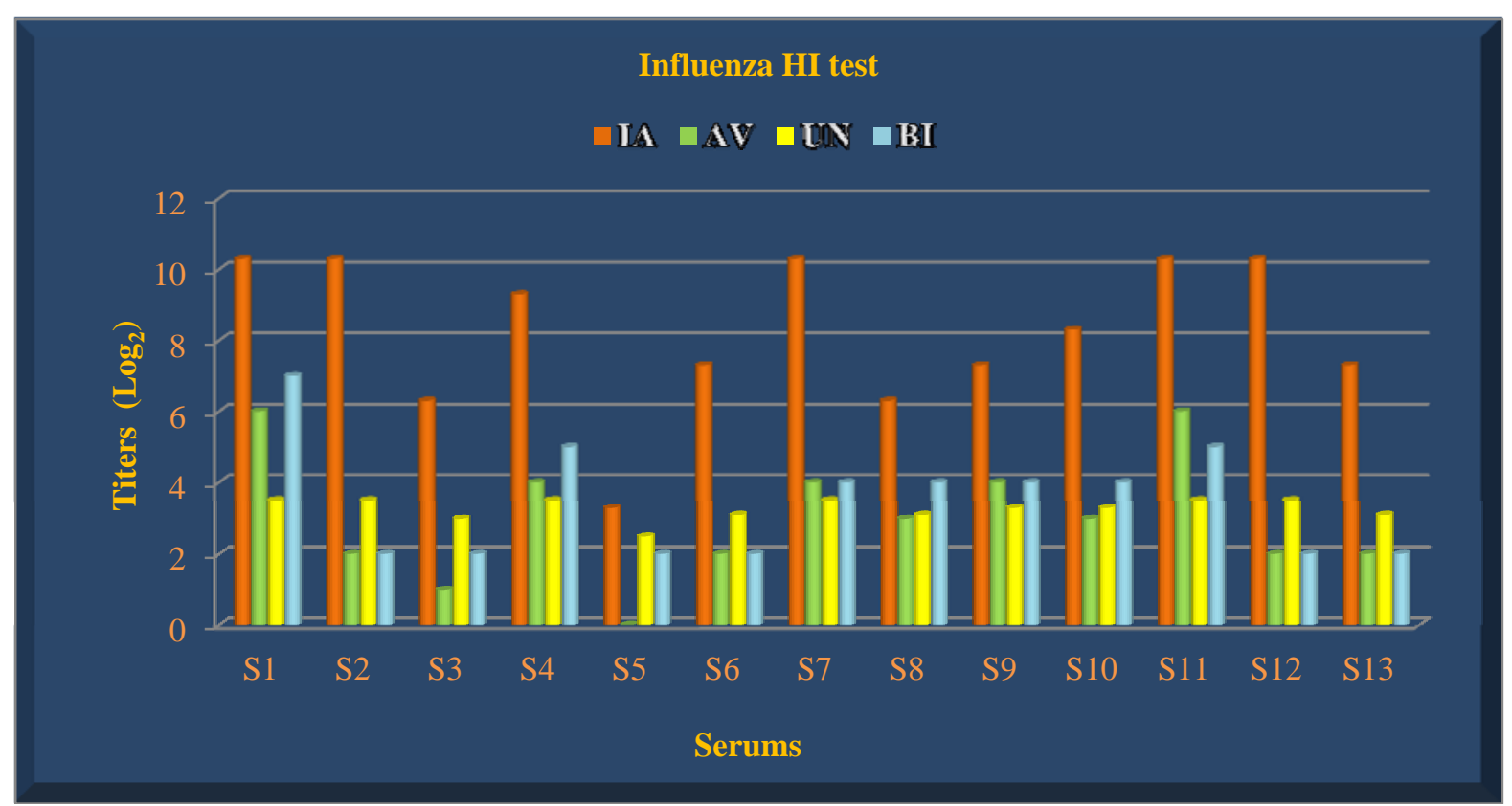

Fig. 6 HI test results of avian influenza of the third serologic sampling at 7-week-old.

(34\% of the flock). According to this, most of the samples analyzed in the laboratory identified as AV in the third sampling would be considered as non-protective and the others (IA, UN and BI) would be considered as protective.

Due to the differences shown in the results obtained by some laboratories, a potency-challenge test should be performed in order to evaluate the real protection against Newcastle disease.
This study matches with the results reported by Abdel-Rhman [18] in 2013, in which a study performed in quails showed that the NDV antibody titers were higher at 0,14 and $28 \mathrm{~d}$ after vaccination in quails vaccinated with the same strain as the antigen used in the test. Whereas, lower antibody titers were obtained when the antigen used in HI test was heterologous [18]. This could explain the results variation. 


\section{Conclusions}

The different laboratories reported very similar results during the first monitoring of the $\mathrm{HI}$ test in both Newcastle disease and avian influenza, while during the second and third monitoring, the results were similar for the HI test for Newcastle disease. The results of the HI test for avian influenza in the second monitoring were very similar in all the laboratories, except in the group identified as IA, where the titers were superior in $92.3 \%$ of the serums.

According to the results obtained in the present study, it can be concluded that the HI test results were expressed according to criteria of the person performing the test. There can be significant differences in logarithms when the same sample is tested in different laboratories. When applying the $4 \times$ 3 factorial with a $95 \%$ confidence, it was statistically determined that the evaluated results were influenced by the laboratory and time.

\section{Acknowledgments}

The authors would like to thank Dr. Gabriela Espinosa Lopez as well as Sanfer-Investigación Aplicada S.A. de C.V. Laboratory for the support given to the accomplishment and publication of this article.

\section{References}

[1] Alexander, D. J., and Senne, D. A. 2008. "Newcastle Disease, Other Avian Paramyxoviruses, and Pneumovirus Infections.” In Diseases of Poultry, 12th ed., edited by Saif, Y. M., Fadly, A. M., Glisson, J. R., McDougald, L. R., Nolan, N. K., and Swayne, D. E. Ames, IA: Blackwell, 75-115.

[2] Seal, B. S., King, D. J., and Sellers, H. S. 2000. "The Avian Response to Newcastle Disease Virus.” Develop. Comp. Immunol. 24: 257-68.

[3] Bennejean, G. 1988. "Newcastle Disease: Control Policies.” In Newcastle Disease, edited by Alexander, D. J. Boston: Kluwer Academic Publishers, 303-17.

[4] Lancaster, J. E. 1976. "A History of Newcastle Disease with Comments on Its Economic Effects." World's Poultry Sci. J. 32 (2): 167-75.

[5] Kaleta, E. F., and Baldauf, C. 1998. "Newcastle Disease in Free-Living and Pet Birds.” In Newcastle Disease, edited by Alexander, D. J. Boston: Kluwer Academic Publishers, 197-246.

[6] Wobeser, G., Leighton, F. A., Norman, R., Myers, D. J., Onderka, D., Pybus, M. J., Neufeld, J. L., Fox, G. A., and Alexander, D. J. 1993. "Newcastle Disease in Wild Water Birds in Western Canada.” Can. Vet. J. 34 (6): 353-9.

[7] Cardenas, G. S., Navarro, L. R., Morales, R., Olvera, M. A., Marquez, M. A., Merino, R., Miller, P. J., and Alfonso, C. L. 2013. "Molecular Epidemiology of Newcastle Disease in Mexico and the Potential Spillover of Viruses from Poultry into Wild Species.” Appl. Environ. Microbiol. 79 (16): 4985-92.

[8] Marangon, S., and Busani, L. 2006. "The Use of Vaccination in Poultry Production.” Rev. Sci. Tech. Off. Int. Epiz. 26 (1): 265-74.

[9] Van Boven, M., Bouma, A., Fabri, T. H. F., Katsma, E., Hartog, L., and Koch, G. 2008. "Herd Immunity to Newcastle Disease Virus in Poultry by Vaccination.” Avian Pathol. 37 (1): 1-5.

[10] World Organization for Animal Health (OIE). 2008. Manual of Diagnostic Tests and Vaccines for Terrestrial Animals (Mammals, Birds and Bees), 6th ed.. Vol. 2. Paris, France: OIE.

[11] Lamb, R. A., and Krug, R. M. 1996. “Orthomyxoviridae: The Viruses and Their Replication.” In Fields Virology, edited by Knipe, D. M., Howle, P. M., and Fields, B. N. Philadelphia: Lippincott-Raven Press, 1487-532.

[12] Capua, I., and Marangon S. 2006. "Control of Avian Influenza in Poultry.” Emerg. Infect. Dis. 12 (9): 1319-24.

[13] Lee, C. W., and Suarez, D. L. 2005. “Avian Influenza Virus: Prospects for Prevention and Control by Vaccination.” Animal Health Res. Rev. 6 (1): 1-15.

[14] World Organization for Animal Health (OIE). 2016. "Avian Influenza (Infection with Avian Influenza Viruses)." In Manual of Diagnostic Tests and Vaccines for Terrestrial Animals 2016. Vol. 1. Paris, France: OIE.

[15] NOM-ZOO. 1997. "Mexican Official Norm, Minimum Requirements for Vaccines Used in Prevention and Control of Newcastle Disease.” NOM-052-ZOO-1995. Accessed April 24, 1997. http://legismex.mty.itesm.mx/normas/zoo/zoo052.pdf.

[16] Valladares de la Cruz, J. C. 2012. "Influenza Avian Diagnosis with ELISA Test from Biochek UK Ltd..” Accessed July 2012. http://www.porcicultura.com/porcicultura/home/impresio n.asp?cve_art=908. (in Spanish)

[17] Kapczynski, D. R., and King, D. J. 2005. "Protection of Chickens against over Clinical Disease and Determination of Viral Shedding Following Vaccination with Commercially Available Newcastle Disease Virus Vaccines upon Challenge with Highly Virulent Virus 
from the California 2002 Exotic Newcastle Disease Outbreak.” Vaccine 23: 3424-33.

[18] Abdel-Rhman, S. S., Al-Jassem, A. H., Hussein, G. M., and Al-Blowi, M. H. 2013. "Comparison between
Haemagglutination Inhibition Test and Enzyme Linked Immune Sorbent Assay in Evaluation of Newcastle Disease Antibodies in Japanese Quails.” J. World's Poult. Res. 3 (3): 83-8. 